\title{
Process improvement based on an integrated approach of DMAIC and multi-method simulation
}

\author{
A. Ahmed, J. Page and J. Olsen \\ NSW University, Sydney, NSW, 2052 \\ The School of Mechanical and Manufacturing Engineering, University of New South Wales, \\ Sydney, Australia. \\ Email: A.F.Ahmed@unsw.edu.au.
}

\begin{abstract}
Many companies have used simulation techniques to improve their operations and generate process improvements for many years. Six Sigma has been developed as a disciplined approach and has introduced an alternative way of thinking about product or process improvement. The concept of combining the advantages of simulation techniques and Six Sigma's methodology has recently led companies and academics to investigate and implement this strategy. The Six Sigma method, composed by the Define, Measure, Analyze, Improve and Control stages, is a problem-solving method. However, it also identifies problems for which the approach may be ineffective. The objective of this study is to improve and control processes, reduce non-value-added activities and support decision-making by using Six Sigma's methodology alongside AnyLogic; using agent-based, discrete event, and system dynamics models. Moreover, the paper explores a multi-method simulation as a guided tool to assist organizations with the decision to implement a Six Sigma approach and pinpoints the strategies to be adjusted and monitor the process' parameters to be improved as well as reduce bottlenecks and weaknesses in the entire process by setting up management guidelines of such complex and dynamic process. This ensures that existing systems and proposed improvements account for any short and long-term outcomes. This paper begins with an overview of Six Sigma, followed by a description and the benefits of using the AnyLogic simulation package for implementing this methodology. This paper also shows the fundamental relationships between the Six Sigma methodology and AnyLogic simulation displaying a framework in which they can be integrated and a business case where this framework is used. The improvement and the strengths from this combination between Six Sigma and simulations are represented as preferable and capable enhancements to Six Sigma to deal with defects in many aspects.
\end{abstract}

Keywords: Six Sigma, DMAIC methodology, agent based, discrete event, system dynamics 


\section{INTRODUCTION}

Increasing competition in many areas such as health care, supply chain operations and manufacturing are forcing managers to reconsider how they manage and control their delivery systems so as to provide excellent and highquality outcomes at reasonable costs. Variations in processes decreases quality and increases costs. One of the main goals of the processes must be to identify and decrease the root causes of variations. Six Sigma is a datadriven, customer-focused, and project-based approach (Black and Revere, 2006) that makes the use of a systematic and structured methodology that depends on DMAIC (Define, Measure, Analyse, Improve, Control). This can generate a roadmap to continuous improvement and thus improve the capability of business processes (El-Haik and Al-Aomar, 2006). To be successfully applied, this methodology necessitates committed, well trained and experienced technical project leadership that can act as a dedicated and competent multifunctional project team (Goldstein, 2001). The use of simulations is one of the several tools which can be used with Six-Sigma (Pyzdek and Keller 2014). Simulation technology has progressed enormously in recent times and various new Software packages are continually coming into the market, while already existing Software packages are being improved and updated. Six-Sigma professionals have difficulty keeping up with this progress. Simulations employ a wide variety of algorithms; the most commonly used to explain business processes and systems are System Dynamics (SD), Agent-Based (AB) and Discrete-Events (DE). Each has particular strengths and implementation procedures. Within simulations, many techniques have been developed that can support the DMAIC implementation in a SixSigma project. This paper also explores the process improvement tools of multi-method simulation techniques $(\mathrm{SD}, \mathrm{AB}$, and $\mathrm{DE})$ to examine the effect of any change in the process' inputs hopefully to improve quality, timeliness and cost. One purpose of this paper is to provide additional quantitative evidence of the benefits of implementing multi-method simulation techniques along with DMAIC stages in the processes. The other purpose is to answer the business research question as to whether the implementation of multi-method simulation techniques with DMAIC stages can improve quality and efficiency of the services in the systems. The rest of the paper is organized as follows: a discussion of six sigma and its methodology (DMAIC). Then a discussion of the Anylogic package and the simulation paradigms (DE, AB, SD) is presented. Following this, we include a major discussion on how these models fit and benefit DMAIC stages, including a business case study. Finally, conclusions and recommendations are provided.

\section{SIX SIGMA}

Six Sigma has been applied globally for many years successfully making significant savings to the bottom-line of many organizations (Treichler, 2005). It is a data-driven and systematic approach to process improvement which aims for the near-elimination of defects from every process, product and transaction (Firka, 2010; Montgomery, 2010). This approach has been broadly used to reduce costs and improve performances in several industrial areas (Kaushik et al., 2012). However, the target of the methodology is not only to improve the process but also to ensure that continuous improvements are made (Snee, 2004). The application of Six Sigma is growing and moving from industrial areas to incorporate business operations like services, administration, sales and marketing and particularly to the areas which affect customers directly (Hahn et al., 2000). It begins with a business strategy and finishes with general-particular implementation and it will have a significant impact on revenue where successfully deployed (Breyfogle, 2003). As a project driven administration approach, the scope of Six Sigma applications is additionally developing from reduction of defects in an organization's processes, items and administrations. It is becoming noticeably, a business methodology that focuses on enhancing comprehension of customer requirements, business efficiency and financial performance (Kwak and Anbari, 2006; Heavey and Murphy, 2012). Despite the fact that Six Sigma begun in the manufacturing industry, nowadays it is being applied in a variety of fields in various sizes of projects and dealing with various sorts of business and process problems (Tjahjono et al., 2010; Julien and Holmshaw, 2012; Deshmukh and Chavan, 2012). There has been considerable further research carried out in different parts of the Six Sigma execution, including examination of this technique with different activities and coordinating the Six Sigma philosophy with different activities (Chiarini, 2011; Jeyaraman and Teo, 2010; Lagrosen et al., 2011; Leon et al., 2012). The DMAIC structure of the Six Sigma approach has been well established as a benchmark tool to the process improvement and customer satisfaction (Chen et al., 2005; Snee, 2010). DMAIC stages are describing as following (De Mast and Lokkerbol 2012): define the objectives of the improvement activity, measure the existing process, analyse the process to identify ways to reduce the gap between the existing performance of the process and the desired and preferred aims, improve the process and control the process.

The cycle of process improvement (DMAIC) starts with defining a problem, the next step is doing the measurement of baseline performance. Appropriate target variables are later identified against possible predictors using proper analytical tools to find out the enhanced process settings. Ultimately, the enhanced process 
performance will be maintained to apply effective control plans. The framework of Six Sigma (DMAIC) uses different non-statistical and statistical tools and techniques to reduce the variation in the process in a controlled manner (Gijo et al., 2011a, 2011b).

\section{ANYLOGIC SIMULATIONS}

Anylogic $\AA$ is a software package designed for simulation tasks by XJ Technologies in 1992. The software includes several types of simulation approaches like DE, SD and AB (Figure 1). All the simulation approaches are managed by using one modelling language and one development environment. The simulation system uses Java language, and the reason that it is effectively flexible for users to represent the heterogeneity and complexity of business, economic and social systems. Also, the details can be changed to correspond to several simulation requirements. This multi-method simulation strengthens the objectoriented model design paradigm that generates possible modular and/or gradual construction of large models. Also, the agents are developed and their behaviour controlled

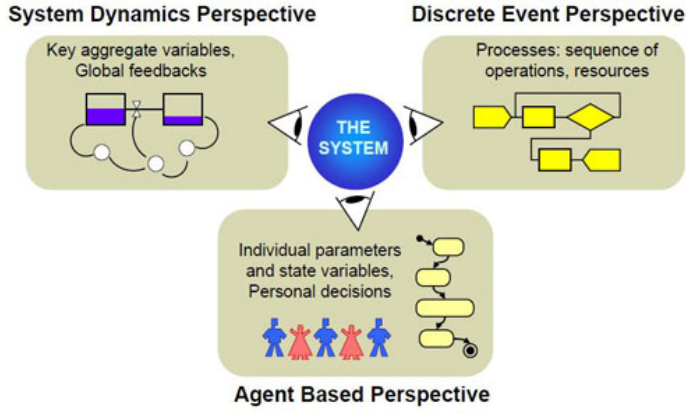

Figure 1. Multi-method model structure (XJ Technologies, n.d). according to their individual built-in state chart. The unique advantage of this simulation package is that it is the only tool that supports DE, AB and SD Simulations. Furthermore, it can run the process in either real time or virtual time for most simulations.

\subsection{System Dynamics Simulations}

Maidstone (2012) presents an SD model as an approach that borrows from the DE variant to provide a distinct presentation of the relationships between the system components and the flow of resources and information between them. The model places a high emphasis on these flows along with inventory quantity and delays in their operations. The SD model's availability allows the user to define the flow of resources, products, and information among the discrete components, which can include a manufacturing line. In this strategy, the company is able to increase its ability to identify the effect of the delays on its overall competitiveness by comparing the results of the simulation with past records. Moreover, Maidstone (2012) mentioned that the SD model also accommodates delays as a critical aspect of its modelling approach, so increasing its capacity to benefit the company's system. Moreover, its ability to predict the system's behavior through a structural analysis of the developed model also enhances its ability to improve the outcomes. This further improves the AnyLogic simulations and their capacity to predict the system's outcomes after implementing any suggested changes. According to a XJ Technologies (n.d.) report, the SD approach has its roots in the fields of science and engineering and thereby strives to analyze systems' flows and the behaviors of the components within these closed systems which makes this approach dependent on the feedback loops to define causality and predict results. Moreover, this method supersedes the DE approach since it allows for the identification of key dependencies for circular causalities where the results of individual feedback loops can allow for an inclusion of vital information sources in the subsequent models (Gunes 2014). This also allows it to have a high level of abstraction due to its capacity to compartmentalize the individual components within the system and describe their behaviors as flows from one component to the next without intrinsically analyzing their motivations. However, this also reduces the ability of SD simulations to be used to understand the case-specific behavior as it appears in the real world, such as morality and other factors that influence an individual agents' behaviors.

\subsection{Discrete-Event Simulations}

In the industrial landscape, the optimization of internal processes for the purposes of improved outcomes requires the operators to understand the components that comprise their systems along with their impact on the operations of the overall system. The use of AnyLogic software in this case will utilize the DE simulation approach, whereby the main target of this approach is its capacity to influence the changes made to the production processes or their outputs (Armijo and Sorli 2014). Lin (2013) also points out that this approach has value in industrial and military applications, where the identification of definitive outcomes is critical in ensuring the maintenance of control over these processes and their outputs. Moreover, optimizing resource utilization is a key benefit of adopting this approach, and it enhances the ability of the operators in question to model and improve the queuing process through an analysis of the possible ramifications that follow each set of decisions. For DE simulations, the 
abstraction extends towards defining the systems under review as processes, thereby allowing an analysis that perceives the entities involved as well as the operations that they conduct and facilitate (XJ Technologies n.d.). In these models, the description of operations extends to the inclusion of delays, as the individual components compete for the resources available. As a result, this approach also enables operators to represent their systems as flow charts, thereby allowing them to determine the points at which the processes begin and terminate. This means that the DE approach differs from SD modelling since it has a lower level of abstraction which reduces the system to a series of steps that the operators can then simulate through sequential process models (XJ Technologies n.d.). Moreover, this approach reduces the entities to passive participants in their systems since they are only source or destination entities that exist for the purpose of carrying data without deterministic analysis of their behaviors or the causalities of their actions.

\subsection{Agent-Based Simulations}

Aside from simulating DE, the AnyLogic software also allows the operators to develop a keener understanding of the entities that depend on and influence their choice of production strategies. For this activity, the AB model enhances the operators' capacity to quantify the factors leading to the current production strategy. Moreover, the model will also enhance the operators' understandings of exiting agents rather than the design of new ones, thereby enhancing the company's understandings of the need to maximize economic benefit across its operations. In this way, the use of the Six Sigma methodology to describe and define these agents and their relationships will benefit from the inclusion of AnyLogic simulation software by allowing the company to identify possible incompatibilities that may result from any changes that they make to the current production strategy. Gunes (2014) notes that $\mathrm{AB}$ modelling is a recent entrant into the field of system modelling and it satisfies the need for deeper system insights that is lacking in the traditional SD and DE approaches. This method also corresponds to improvements in the field of computer science, which include the Unified Modelling Language (UML) as well as the exponential improvements in computing power that introduced the ability to engage in more complex computations. This approach acknowledges that the system's components may also have their individual behaviors that influence their interactions with the system, thereby requiring operators to enable these agents to interact within their environments and with other components that have dynamic behavior. As a result, this approach generates a more naturalistic understanding of systems compared to the more methodical strategies in SD and DE modelling. This allows it to model systems into state charts that individuals progress through if they invoke particular transitional triggers (XJ Technologies n.d.). The ability to model asynchronous events and interactions allows the $\mathrm{AB}$ approach to exist at any level of abstraction, thereby delimiting it and improving its capacity to develop deterministic simulations of these individual entities' behaviors more accurately.

\section{COMBINING SIX SIGMA AND SIMULATIONS}

The Six Sigma methodology is the result of repeated attempts across organizations to improve their ability to predict the impact of their actions on the outcomes of their systems. The addition of AnyLogic allows the management elements to simulate their systems, and predict the influence that changes to the system would have on the outcomes. However, it is also vital to note that AnyLogic allows for extended simulations, whereby the virtual environments can produce results spanning several years. With this functionality, it becomes possible to determine the impact of their decisions on the short and long-term process outputs. According to Taneja and Manchanda (2013), this also allows Six Sigma models to improve their awareness of the interactions between system components as well as their significance in the overall system. Considering the long-term implications of manufacturing waste, this approach improves the ability of management to make informed decisions. Therefore, the combination of Six Sigma modelling and multi-method simulations is an essential improvement upon existing approaches and enhances the ability of management to prioritize key decisions for improved short and long-term efficiency.

\section{A FRAMEWORK TO INTEGRATE MULTI-METHOD SIMULATIONS INTO THE DMAIC METHODOLOGY}

The use of Six Sigma is well documented, with Hahn, et al, (2000), Raisinghani (2005) and Deshmukh and Chavan (2012), providing examples of its use and efficacy across industrial use cases. The use of Six Sigma's DMAIC methodology alongside AB, DE and SD models aims to improve the quality of process improvement efforts targeted at enhancing productivity and reducing waste. This paper analyzes the efficacy of simulation tools as an addition to the modeling frameworks of the Six Sigma methodology as a means of improving its ability to analyze and model complex production environments. There are various simulation approaches that can be used for engaging in these process improvement exercises, and this research studies them to define a framework in which to work. 
In the define stage of DMAIC, SD provides powerful tools, such as causal loop diagrams and group model building to determine internal and external elements that help understand the key drivers that influence the desired behavior on a factory environment. Using the very same tools to abstract system components, SD can be fundamental to define resource and information flows from a qualitative perspective and set up the starting point for a six-sigma project roadmap and the high-level strategy following the DMAIC methodology.

The measure phase focuses on understanding how the system or process associated to the defined problem works, including the identification of inputs and outputs, causal relationships and the interaction of internal variables. In this phase, the most suitable simulation method depends on the problem at hand. For a clearly defined manufacturing flowchart, a DE model will be the best tool to explain variation of products, bottlenecks and resource utilization. For a high level SIPOC (Supplier, Input, Process, Output, Customer) table or a long-term business strategy, SD will provide the necessary abstraction to generate a well-defined model, but for human or machine interactions, $\mathrm{AB}$ models have the necessary elements to understand the complex granular outcomes that can arise.

The analyze phase has the objective of processing the data to come up with potential solutions. A DE model will go through statistical analysis to understand bottlenecks, process variations or resource utilization. A SD model will go through structural validations, parameter variation experiments, Monte Carlo simulations and comparative runs with a reference mode if there is one. The variety of AB models is vast and they are analyzed with statistical methods using hypothesis testing, analysis of variance or covariance and regression analysis among others. Hybrid models will use a combination of these analysis techniques.

The improve phase will put in place the new strategy that was discovered during the analyze phase using any of the three simulation methods or hybrids. A change in a process will add a DE model or update the DE model generated during the measure phase generally through the use of flowcharts. Changes in strategic decisions or high level processes will add the SD paradigm to the model or update the SD generated during the measure phase with new feedback loops or new policy structures. A change in human or machine behavior or the addition of new entities to the system will add a new $\mathrm{AB}$ model or update the $\mathrm{AB}$ model presented in the measure phase to take these new elements into consideration. Hybrid combinations may be added where the improvement project requires many types of changes.

Finally, the control phase will test the long-term behavior of the solution using simulations while never touching the production system until everything is tested and validated. As highlighted by Madistone (2012), the decision on what simulation method to use is contextual and needs to be re-evaluated in each step of the DMAIC process in order to continue with the same method or use multi-method techniques to integrate new improvement strategies. In this way, AnyLogic provides a vital contribution to the utility of Six Sigma's DMAIC methodology by allowing the simulation of both static and dynamic resources within the analyzed system. Additionally, AnyLogic is vital in benchmarking the results of improvement attempts due to its provisions for long-term simulations. In particular, this allows for extended analyses of the impact that changes to various elements and flows have on system outputs, while also incorporating the unique characteristics and decision-making behaviors of individual agents. This shows that these three simulation approaches are essential enhancements to the DMAIC methodology, and that their inclusion as part of AnyLogic enhances its utility in improving the ability to increase productivity while reducing waste.

\section{BUSINESS CASE - APPLICATION OF THE FRAMEWORK}

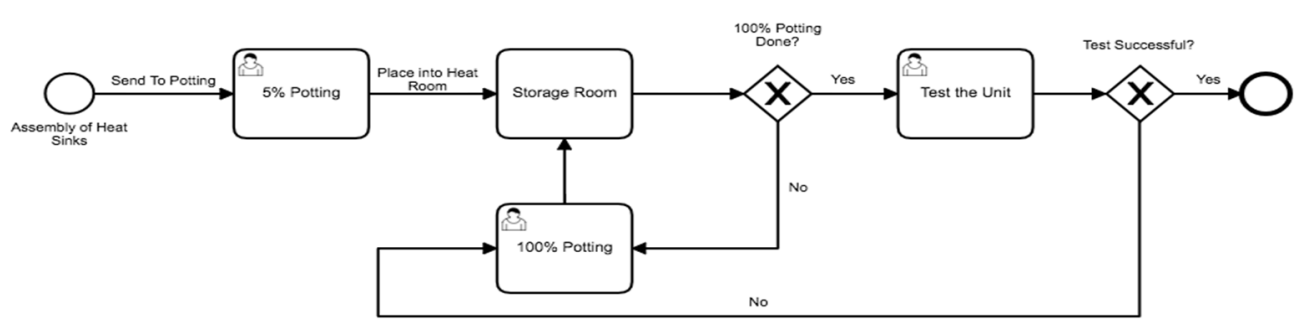

Figure 2. A segment of the LED manufacturing process - Potting

The proposed framework was used in a small LED-manufacturing factory where a multi-method simulation model was developed within the DMAIC context to improve the efficiency of the production process. The study examined whether this novel framework could be used for this purpose, thereby, improving efficiency and effectiveness in a manufacturing setting. It made use of several tools, in successive steps, to accomplish this, including DE, SD and AB computer simulations. AnyLogic was used to model the existing process followed by the implementation of changes in the same platform. The purpose of this Six Sigma project was to find a way to 
reduce manufacturing time in order to increase weekly production and meet the increasing demand. With the problem initially defined, the measure phase consisted on the development of a flowchart that explained the current state of the LED manufacturing process (figure 2) as well as a SIPOC table explaining an external manufacturing process happening outside the LED factory (figure 3). While the flowchart was built in AnyLogic using the DE paradigm, the SIPOC table was represented using SD as it is advised in the proposed framework guideline.

In the analyse phase, an $\mathrm{AB}$ model was added to visualize through the use of animations, the interactions between individual products and the environment, generating this way a multi-method simulation integrating all three paradigms into a unique model. This analysis included:

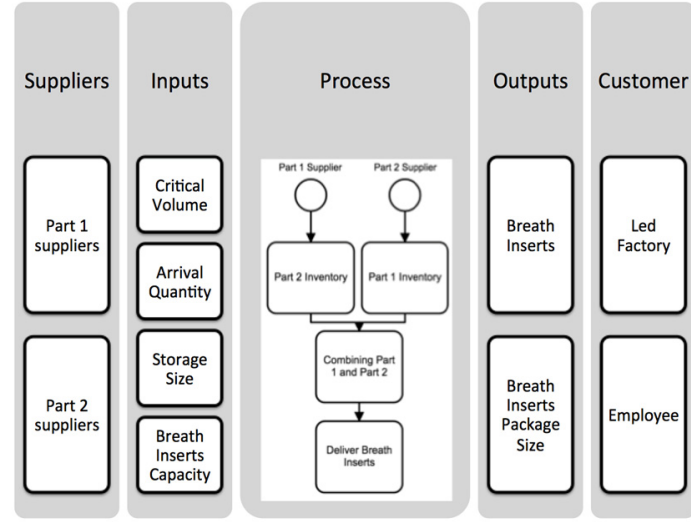

Figure 3. SIPOC table - Breath Inserts variation on raw material delivery, resource utilization, queues, production variations, processing time statistics and financial metrics. This analysis generated a clear potential solution through the discovery of unnecessary inventory waste that created bottlenecks during the process. The improvement proposed in this case study was to have a better coordination between raw material suppliers and operations, requiring a redistribution of human resources and tasks when inventory rises. Since this is a change in the process, and not a strategic policy or a change in the interaction between the entities composing the system, the flowchart was redefined and changes were built in the DE segment of the model. This is aligned not only with the proposed framework guideline, but also with the contextual dependency of the model used. This process change led to an increase in production of $35 \%$ and a reduction of costs per product of around $8 \%$. During the control phase, the long-term simulation didn't seem to show any negative consequences due to changes in the process, validating this way the solution for implementation in the real production line.

Using the DMAIC approach allowed the project to engage in a sequential analysis of the manufacturing processes within the factory as well as their inputs and outputs, providing a more elaborate picture of the factory model. As a result, the success of the simulations is dependent upon the accuracy and efficacy of the developed model, thereby making the Six Sigma methodology an instrumental element in the overall process improvement exercise.

\section{CONCLUSIONS}

As a result, this research counts as a vital source of information for both professionals looking to improve the efficiency of their systems as well as researchers looking to enhance their understandings of this combination of tools as a means of broadening the knowledge base regarding their use. Furthermore, the increasing need for companies to reduce waste due to productivity and environmental concerns drives this research. In enhancing SixSigma methodology, it is essential to raise awareness of simulations and the specific tools available and how to use them independently in the DMAIC stages and generate multi-method or hybrid simulation. These techniques when the context is suitable represent better the interacted processes, separate behaviour and corporate long-term plan or strategy. This paper reviews the application of Six Sigma (DMAIC methodology) and AnyLogic simulation techniques in a manufacturing environment. The objective of the study was to show how we could improve and control processes in manufacturing to achieve reduce costs and increase customer satisfaction. In this respect, the approach of combining Six Sigma and AnyLogic by using the DMAIC method and the applied simulation techniques incorporated in the words Analysis, Improve and Control was explained along with how this could bring huge benefits to reduce defects. So, the AnyLogic simulation is a good fit for DMAIC as part of Design for Six-Sigma in all its five stages. Future work is needed on this topic with more corporate cases that are requiring multi-method modelling especially with DMAIC stages with the intention of further understanding the implications and a new vision with Six-Sigma projects.

\section{REFERENCES}

Armijo, A. and Sorli, M. (2014). Interoperable Process Engineering System for Collaborative Optimization of Product Life-Cycle. Revolutionizing Enterprise Interoperability through Scientific Foundations, pp.166-191.

Black, K. and Revere, L. (2006). "Six Sigma arises from the ashes of TQM with a twist", International Journal of Health Care Quality Assurance, Vol. 19 No. 3, pp. 259-266.

Breyfogle, F.W. (2003). Implementing Six Sigma: Smarter Solutions Using Statistical Methods, John Wiley, New York, NY. 
Chen, S.C., Chen, K.S. and Hsia, T.C. (2005). "Promoting customer satisfactions by applying six sigma: an example from the automobile industry", The Quality Management Journal, Vol. 12 No. 4, pp. 21-33.

Chiarini, A. (2011). "Japanese total quality control, TQM, Deming's system of profound knowledge, BPR, Lean and Six Sigma: comparison and discussion”, International Journal of Lean Six Sigma, Vol. 2 No. 4, pp. 332355.

De Mast, J. and J. Lokkerbol (2012). "An analysis of the Six Sigma DMAIC method from the perspective of problem solving." International Journal of Production Economics 139(2): 604-614.

Deshmukh, S.V. and Chavan, A. (2012). "Six Sigma and SMEs: a critical review of literature", International Journal of Lean Six Sigma, Vol. 3 No. 2, pp. 157-167.

El-Haik, B.S. and Al-Aomar, R. (2006). Simulation-Based Lean Six Sigma and Design for Six Sigma, Wiley, Hoboken, NJ.

Firka, D. (2010). "Six Sigma: an evolutionary analysis through case studies”, The TQM Journal, Vol. 22 No. 4 , pp. 423-434.

Güneş, G. (2014). Agent-Based Simulation and An Example In AnyLogic.

Gijo, E.V. and Scaria, J. (2011a). "Application of Taguchi method to optimise the characteristics of green sand in a foundry", International Journal of Business Excellence, Vol. 4 No. 2, pp. 191-201.

Gijo, E.V., Scaria, J. and Antony, J. (2011b). "Application of Six Sigma methodology to reduce defects of a grinding process”, Quality and Reliability Engineering International, Vol. 27 No. 8, pp. 1221-1234.

Goldstein, M. (2001). "Six Sigma program success factors", Six Sigma Forum Magazine, Vol. 1 No. 1, pp. 3645.

Hahn, G.J., Doganaksoy, N. and Hoerl, R. (2000). The evolution of six sigma. Quality Engineering, 12(3), pp.317326.

Heavey, C. and Murphy, E. (2012). "Integrating the balanced scorecard with Six Sigma", TheTQM Journal, Vol. 24 No. 2, pp. 108-122.

Jeyaraman, K. and Teo, L.K. (2010). "A conceptual framework for critical success factors of lean Six Sigma: implementation on the performance of electronic manufacturing service industry", International Journal of Lean Six Sigma, Vol. 1 No. 3, pp. 191-215.

Julien, D. and Holmshaw, P. (2012). "Six Sigma in a low volume and complex environment", International Journal of Lean Six Sigma, Vol. 3 No. 1, pp. 28-44.

Kaushik, P., Khanduja, D., Mittal, K. and Jaglan, P. (2012). "A case study: application of Six Sigma methodology in a small and medium-sized manufacturing enterprise", The TQM Journal, Vol. 24 No. 1, pp. 4-16.

Kwak, Y.H. and Anbari, F.T. (2006). "Benefits, obstacles and future of Six Sigma approach", Technovation, Vol. 26 Nos. 5/6, pp. 708-715.

Lagrosen, Y., Chebl, R. and Tuesta, M.R. (2011). "Organisational learning and Six Sigma deployment readiness evaluation: a case study”, International Journal of Lean Six Sigma, Vol. 2 No. 1, pp. 23-40.

Leon, H.C.M., Perez, M.D.C.T., Farris, J.A. and Beruvides, M.G. (2012). "Integrating Six Sigma tools using team-learning processes", International Journal of Lean Six Sigma, Vol. 3 No. 2, pp. 133-156.

Lin, M.S. (2013). The Research of Quality Prediction Model for Touch Panel Manufacturing.

Maidstone, R. (2012). "Discrete Event Simulation, System Dynamics and Agent Based Simulation : Discussion and Comparison." System 1-6.

Pyzdek, T., \& Keller, P. A. (2014). The six sigma handbook: McGraw-Hill Education.

Raisinghani, M.S. (2005). "Six Sigma: concepts, tools, and applications", Industrial Management and Data Systems, Vol. 105 No. 4, pp. 491-505.

Snee, R.D. (2004). "Six Sigma: the evolution of 100 years of business improvement methodology", International Journal of Six Sigma and Competitive Advantage, Vol. 1 No. 1, pp. 4-20.

Snee, R.D. (2010). "Lean Six Sigma-getting better all the time", International Journal of Lean Six Sigma, Vol. 1 No. 1, pp. 9-29.

Tjahjono, B., Ball, P, Vitanov, V.I., Scorzafave, C., Nogueira, J., Calleja, J., Minguet, M., Narasimha, L., Rivas, A., Srivastava, A., Srivastava, S. and Yadav, A. (2010). "Six Sigma: a literature review", International Journal of Lean Six Sigma, Vol. 1 N. 3, pp. 216-233.

Taneja, M. and Manchanda, A. (2013). Six sigma an approach to improve productivity in manufacturing industry. International Journal of Engineering Trends and Technology (IJETT), 5(6), pp.281-286.

Treichler, D.H. (2005). The Six Sigma Path to Leadership, Pearson Education, Delhi.

XJ Technologies Company (1992), available from: www.xjtek.com. 